PROCEEDINGS OF THE

AMERICAN MATHEMATICAL SOCIETY

Volume 132, Number 5, Pages 1279-1283

S 0002-9939(03)07466-5

Article electronically published on November 7, 2003

\title{
RINGS WITH FINITE GORENSTEIN INJECTIVE DIMENSION
}

\author{
HENRIK HOLM \\ (Communicated by Bernd Ulrich)
}

\begin{abstract}
In this paper we prove that for any associative ring $R$, and for any left $R$-module $M$ with finite projective dimension, the Gorenstein injective dimension $\operatorname{Gid}_{R} M$ equals the usual injective dimension $\operatorname{id}_{R} M$. In particular, if $\operatorname{Gid}_{R} R$ is finite, then also $\mathrm{id}_{R} R$ is finite, and thus $R$ is Gorenstein (provided that $R$ is commutative and Noetherian).
\end{abstract}

\section{INTRODUCTION}

It is well known that among the commutative local Noetherian rings $(R, \mathfrak{m}, k)$, the Gorenstein rings are characterized by the condition $\operatorname{id}_{R} R<\infty$. From the dual of [10, Proposition (2.27)] ([6, Proposition 10.2.3] is a special case) it follows that the Gorenstein injective dimension $\operatorname{Gid}_{R}(-)$ is a refinement of the usual injective dimension $\operatorname{id}_{R}(-)$ in the following sense:

For any $R$-module $M$ there is an inequality $\operatorname{Gid}_{R} M \leqslant \operatorname{id}_{R} M$, and if $\operatorname{id}_{R} M<\infty$, then there is an equality $\operatorname{Gid}_{R} M=\operatorname{id}_{R} M$.

Now, since the injective dimension $\operatorname{id}_{R} R$ of $R$ measures Gorensteinness, it is only natural to ask what does the Gorenstein injective dimension $\operatorname{Gid}_{R} R$ of $R$ measure? As a consequence of Theorem (2.1) below, it turns out that:

An associative ring $R$ with $\operatorname{Gid}_{R} R<\infty$ also has $\operatorname{id}_{R} R<\infty$ (and hence $R$ is Gorenstein, provided that $R$ is commutative and Noetherian).

This result is proved by Christensen [2, Theorem (6.3.2)] in the case where $(R, \mathfrak{m}, k)$ is a commutative local Noetherian Cohen-Macaulay ring with a dualizing module. The aim of this paper is to prove Theorem (2.1), together with a series of related results. Among these results is Theorem (3.2), which has the nice, and easily stated, Corollary (3.3):

Assume that $(R, \mathfrak{m}, k)$ is a commutative local Noetherian ring, and let $M$ be an $R$-module of finite depth, that is, $\operatorname{Ext}_{R}^{m}(k, M) \neq 0$ for some $m \in \mathbb{N}_{0}$ (this happens for example if $M \neq 0$ is finitely generated). If either

(i) $\operatorname{Gfd}_{R} M<\infty$ and $\operatorname{id}_{R} M<\infty$ or $($ ii $) \operatorname{fd}_{R} M<\infty$ and $\operatorname{Gid}_{R} M<\infty$, then $R$ is Gorenstein.

Received by the editors January 28, 2003.

2000 Mathematics Subject Classification. Primary 13D02, 13D05, 13D07, 13H10; Secondary 16E05, 16E10, 16E30.

Key words and phrases. Gorenstein dimensions, homological dimensions, Gorenstein rings. 
This corollary is also proved by Christensen [2, Theorem (6.3.2)] in the case where $(R, \mathfrak{m}, k)$ is Cohen-Macaulay with a dualizing module. However, Theorem (3.2) itself (dealing not only with local rings) is a generalization of [8, Proposition 2.10] (in the module case) by Foxby from 1979.

We should briefly mention the history of Gorenstein injective, projective and flat modules: Gorenstein injective modules over an arbitrary associative ring, and the related Gorenstein injective dimension, was introduced and studied by Enochs and Jenda in [3]. The dual concept, Gorenstein projective modules, was already introduced by Auslander and Bridger [1] in 1969, but only for finitely generated modules over a two-sided Noetherian ring. Gorenstein flat modules were also introduced by Enochs and Jenda; please see [5].

1.1. Setup and notation. Let $R$ be any associative ring with a nonzero multiplicative identity. All modules are -if not specified otherwise - left $R$-modules. If $M$ is any $R$-module, we use $\operatorname{pd}_{R} M, \operatorname{fd}_{R} M$, and $\operatorname{id}_{R} M$ to denote the usual projective, flat, and injective dimension of $M$, respectively. Furthermore, we write $\operatorname{Gpd}_{R} M, \operatorname{Gfd}_{R} M$, and $\operatorname{Gid}_{R} M$ for the Gorenstein projective, Gorenstein flat, and Gorenstein injective dimension of $M$, respectively.

\section{Rings With Finite GoRenstein injeCtive Dimension}

Theorem 2.1. If $M$ is an $R$-module with $\operatorname{pd}_{R} M<\infty$, then $\operatorname{Gid}_{R} M=\operatorname{id}_{R} M$. In particular, if $\operatorname{Gid}_{R} R<\infty$, then also $\operatorname{id}_{R} R<\infty$ (and hence $R$ is Gorenstein, provided that $R$ is commutative and Noetherian).

Proof. Since $\operatorname{Gid}_{R} M \leqslant \operatorname{id}_{R} M$ always, it suffices to prove that $\operatorname{id}_{R} M \leqslant \operatorname{Gid}_{R} M$. Naturally, we may assume that $\operatorname{Gid}_{R} M<\infty$.

First consider the case where $M$ is Gorenstein injective, that is, $\operatorname{Gid}_{R} M=0$. By definition, $M$ is a kernel in a complete injective resolution. This means that there exists an exact sequence $\boldsymbol{E}=\cdots \rightarrow E_{1} \rightarrow E_{0} \rightarrow E_{-1} \rightarrow \cdots$ of injective $R$-modules, such that $\operatorname{Hom}_{R}(I, \boldsymbol{E})$ is exact for every injective $R$-module $I$, and such that $M \cong \operatorname{Ker}\left(E_{1} \rightarrow E_{0}\right)$. In particular, there exists a short exact sequence $0 \rightarrow M^{\prime} \rightarrow E \rightarrow M \rightarrow 0$, where $E$ is injective, and $M^{\prime}$ is Gorenstein injective. Since $M^{\prime}$ is Gorenstein injective and $\operatorname{pd}_{R} M<\infty$, it follows by [4, Lemma 1.3] that $\operatorname{Ext}_{R}^{1}\left(M, M^{\prime}\right)=0$. Thus $0 \rightarrow M^{\prime} \rightarrow E \rightarrow M \rightarrow 0$ is split-exact; so $M$ is a direct summand of the injective module $E$. Therefore, $M$ itself is injective.

Next consider the case where $\operatorname{Gid}_{R} M>0$. By [10, Theorem (2.15)] there exists an exact sequence $0 \rightarrow M \rightarrow H \rightarrow C \rightarrow 0$ where $H$ is Gorenstein injective and $\operatorname{id}_{R} C=\operatorname{Gid}_{R} M-1$. As in the previous case, since $H$ is Gorenstein injective, there exists a short exact sequence $0 \rightarrow H^{\prime} \rightarrow I \rightarrow H \rightarrow 0$ where $I$ is injective and $H^{\prime}$

is Gorenstein injective. Now consider the pull-back diagram with exact rows and 
columns:

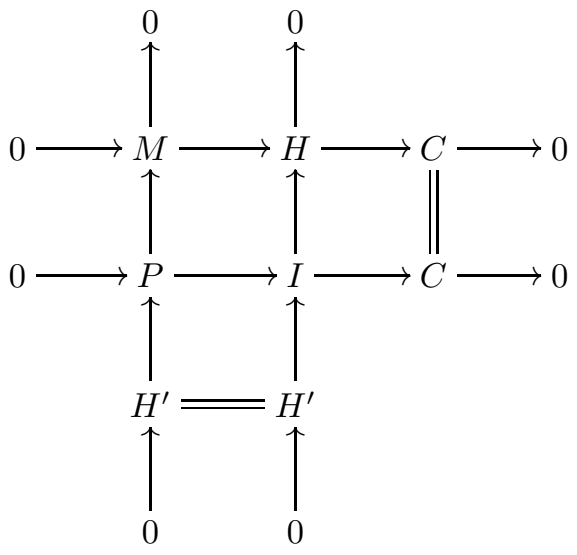

Since $I$ is injective and $\operatorname{id}_{R} C=\operatorname{Gid}_{R} M-1$ we get $\operatorname{id}_{R} P \leqslant \operatorname{Gid}_{R} M$ by the second row. Since $H^{\prime}$ is Gorenstein injective and $\operatorname{pd}_{R} M<\infty$, it follows (as before) by 4 . Lemma 1.3] that $\operatorname{Ext}_{R}^{1}\left(M, H^{\prime}\right)=0$. Consequently, the first column $0 \rightarrow H^{\prime} \rightarrow P \rightarrow$ $M \rightarrow 0$ splits. Therefore $P \cong M \oplus H^{\prime}$, and hence $\operatorname{id}_{R} M \leqslant \operatorname{id}_{R} P \leqslant \operatorname{Gid}_{R} M$.

The theorem above has, of course, a dual counterpart:

Theorem 2.2. If $M$ is an $R$-module with $\operatorname{id}_{R} M<\infty$, then $\operatorname{Gpd}_{R} M=\operatorname{pd}_{R} M$.

Theorem (2.6) below is a "flat version" of the two previous theorems. First recall the following.

Definition 2.3. The left finitistic projective dimension $\operatorname{LeftFPD}(R)$ of $R$ is defined as

$$
\operatorname{LeftFPD}(R)=\sup \left\{\operatorname{pd}_{R} M \mid M \text { is a left } R \text {-module with } \operatorname{pd}_{R} M<\infty\right\} .
$$

The right finitistic projective dimension $\operatorname{RightFPD}(R)$ of $R$ is defined similarly.

Remark 2.4. When $R$ is commutative and Noetherian, we have that $\operatorname{LeftFPD}(R)$ and RightFPD $(R)$ equals the Krull dimension of $R$, by [9, Théorème (3.2.6) (Seconde partie)].

Furthermore, we will need the following result from [10, Proposition (3.11)]:

Proposition 2.5. For any (left) $R$-module $M$ the inequality

$$
\operatorname{Gid}_{R} \operatorname{Hom}_{\mathbb{Z}}(M, \mathbb{Q} / \mathbb{Z}) \leqslant \operatorname{Gfd}_{R} M
$$

holds. If $R$ is right coherent, then we have $\operatorname{Gid}_{R} \operatorname{Hom}_{\mathbb{Z}}(M, \mathbb{Q} / \mathbb{Z})=\operatorname{Gfd}_{R} M$.

We are now ready to state:

Theorem 2.6. For any $R$-module $M$, the following conclusions hold:

(i) Assume that $\operatorname{LeftFPD}(R)$ is finite. If $\mathrm{fd}_{R} M<\infty$, then $\operatorname{Gid}_{R} M=\mathrm{id}_{R} M$.

(ii) Assume that $R$ is left and right coherent with finite $\operatorname{RightFPD}(R)$. If $\operatorname{id}_{R} M<\infty$, then $\operatorname{Gfd}_{R} M=\operatorname{fd}_{R} M$.

Proof. (i) If $\mathrm{fd}_{R} M<\infty$, then also $\operatorname{pd}_{R} M<\infty$, by [11, Proposition 6] (since $\operatorname{LeftFPD}(R)<\infty)$. Hence the desired conclusion follows from Theorem (2.1) above.

(ii) Since $R$ is left coherent, we have that $\operatorname{fd}_{R} \operatorname{Hom}_{\mathbb{Z}}(M, \mathbb{Q} / \mathbb{Z}) \leqslant \operatorname{id}_{R} M<\infty$, by [12, Lemma 3.1.4]. By assumption, $\operatorname{RightFPD}(R)<\infty$, and therefore also 
$\operatorname{pd}_{R} \operatorname{Hom}_{\mathbb{Z}}(M, \mathbb{Q} / \mathbb{Z})<\infty$, by [11, Proposition 6]. Now Theorem (2.1) gives that $\operatorname{Gid}_{R} \operatorname{Hom}_{\mathbb{Z}}(M, \mathbb{Q} / \mathbb{Z})=\operatorname{id}_{R} \operatorname{Hom}_{\mathbb{Z}}(M, \mathbb{Q} / \mathbb{Z})$. It is well known that

$$
\mathrm{fd}_{R} M=\operatorname{id}_{R} \operatorname{Hom}_{\mathbb{Z}}(M, \mathbb{Q} / \mathbb{Z})
$$

(without assumptions on $R$ ), and by Proposition (2.5) above, we also get $\operatorname{Gfd}_{R} M=$ $\operatorname{Gid}_{R} \operatorname{Hom}_{\mathbb{Z}}(M, \mathbb{Q} / \mathbb{Z})$, since $R$ is right coherent. The proof is done.

\section{A theOREM on Gorenstein RINGS By FoXbY}

We end this paper by generalizing a theorem [8, Proposition 2.10] on Gorenstein rings by Foxby from 1979. For completeness, we briefly recall:

3.1. The small support. Assume that $R$ is commutative and Noetherian. For an $R$-module $M$, an integer $n$, and a prime ideal $\mathfrak{p}$ in $R$, we write $\beta_{n}^{R}(\mathfrak{p}, M)$, respectively, $\mu_{R}^{n}(\mathfrak{p}, M)$, for the $n$th Betti number, respectively, $n$th Bass number, of $M$ at $\mathfrak{p}$.

Foxby [8, Definition p. 157] or [7, (14.8)] defines the small (or homological) support of an $R$-module $M$ to be the set

$$
\operatorname{supp}_{R} M=\left\{\mathfrak{p} \in \operatorname{Spec} R \mid \exists n \in \mathbb{N}_{0}: \beta_{n}^{R}(\mathfrak{p}, M) \neq 0\right\} .
$$

Let us mention the most basic results about the small support, all of which can be found in [8, pp. $157-159]$ and [7] Chapter 14]:

(a) The small support, $\operatorname{supp}_{R} M$, is contained in the usual (large) support, $\operatorname{Supp}_{R} M$, and $\operatorname{supp}_{R} M=\operatorname{Supp}_{R} M$ if $M$ is finitely generated. Also, if $M \neq 0$, then $\operatorname{supp}_{R} M \neq \emptyset$.

(b) $\operatorname{supp}_{R} M=\left\{\mathfrak{p} \in \operatorname{Spec} R \mid \exists n \in \mathbb{N}_{0}: \mu_{R}^{n}(\mathfrak{p}, M) \neq 0\right\}$.

(c) Assume that $(R, \mathfrak{m}, k)$ is local. If $M$ is an $R$-module with finite depth, that is,

$$
\operatorname{depth}_{R} M:=\inf \left\{m \in \mathbb{N}_{0} \mid \operatorname{Ext}_{R}^{m}(k, M) \neq 0\right\}<\infty
$$

(this happens for example if $M \neq 0$ is finitely generated), then $\mathfrak{m} \in$ $\operatorname{supp}_{R} M$, by (b) above.

Now, given these facts about the small support, and the results in the previous section, the following generalization of [8, Proposition 2.10] is immediate:

Theorem 3.2. Assume that $R$ is commutative and Noetherian. Let $M$ be any $R$-module, and assume that any of the following four conditions is satisfied:

(i) $\operatorname{Gpd}_{R} M<\infty$ and $\operatorname{id}_{R} M<\infty$,

(ii) $\operatorname{pd}_{R} M<\infty$ and $\operatorname{Gid}_{R} M<\infty$,

(iii) $R$ has finite Krull dimension, and $\operatorname{Gfd}_{R} M<\infty$ and $\operatorname{id}_{R} M<\infty$,

(iv) $R$ has finite Krull dimension, and $\operatorname{fd}_{R} M<\infty$ and $\operatorname{Gid}_{R} M<\infty$.

Then $R_{\mathfrak{p}}$ is a Gorenstein local ring for all $\mathfrak{p} \in \operatorname{supp}_{R} M$.

Corollary 3.3. Assume that $(R, \mathfrak{m}, k)$ is a commutative local Noetherian ring. If there exists an $R$-module $M$ of finite depth, that is,

$$
\operatorname{depth}_{R} M:=\inf \left\{m \in \mathbb{N}_{0} \mid \operatorname{Ext}_{R}^{m}(k, M) \neq 0\right\}<\infty,
$$

and which satisfies either

(i) $\operatorname{Gfd}_{R} M<\infty$ and $\operatorname{id}_{R} M<\infty$, or

(ii) $\operatorname{fd}_{R} M<\infty$ and $\operatorname{Gid}_{R} M<\infty$,

then $R$ is Gorenstein. 


\section{ACKNOWLEDGMENTS}

I would like to express my gratitude to my Ph.D. advisor Hans-Bjørn Foxby for his support, and our helpful discussions.

\section{REFERENCES}

[1] M. Auslander and M. Bridger, Stable module theory, Mem. Amer. Math. Soc. 94, American Mathematical Society, Providence, RI, 1969. MR 42:4580

[2] L. W. Christensen, Gorenstein dimensions, Lecture Notes in Math. 1747, Springer-Verlag, Berlin, 2000. MR 2002e: 13032

[3] E. E. Enochs and O. M. G. Jenda, Gorenstein injective and projective modules, Math. Z. 220 (1995), 611 - 633. MR 97c:16011

[4] E. E. Enochs and O. M. G. Jenda, Gorenstein Balance of Hom and Tensor, Tsukuba J. Math. 19, No. 1 (1995), 1 - 13. MR 97a:16019

[5] E. E. Enochs and O. M. G. Jenda, Gorenstein Injective and Flat Dimensions, Math. Japonica 44, No. 2 (1996), 261 - 268. MR 97k:13019

[6] E. E. Enochs and O. M. G. Jenda, Relative Homological Algebra, de Gruyter Expositions in Math. 30, Walter de Gruyter, Berlin, 2000. MR 2001h:16013

[7] H.-B. Foxby, Hyperhomological Algebra 83 Commutative Rings, notes in preparation.

[8] H.-B. Foxby, Bounded complexes of flat modules, J. Pure and Appl. Algebra 15, No. 2 (1979), 149 - 172. MR 83c:13008

[9] L. Gruson and M. Raynaud, Critères de platitude et de projectivité. Techniques de "platification" d'un module, Invent. Math. 13 (1971), 1 - 89. MR 46:7219

[10] H. Holm, Gorenstein Homological Dimensions, J. Pure and Appl. Algebra (to appear)

[11] C. U. Jensen, On the Vanishing of $\mathrm{lim}^{(i)}$, J. Algebra 15 (1970), 151 - 166. MR 41:5460

[12] J. Xu, Flat covers of modules, Lecture Notes in Math. 1634, Springer-Verlag, Berlin, 1996. MR 98b:16003

Matematisk Afdeling, Københavns Universitet, Universitetsparken 5, 2100 KøbenHAVN $\varnothing$, DANMARK

E-mail address: holm@math.ku.dk 